\title{
BMJ Global Health Pharmaceutical quality assurance of local private distributors: a secondary analysis in 13 low-income and middle- income countries
}

\author{
Kerlijn Van Assche, ${ }^{1}$ Ariadna Nebot Giralt, ${ }^{1}$ Jean Michel Caudron, ${ }^{2}$ \\ Benedetta Schiavetti, ${ }^{3}$ Corinne Pouget, ${ }^{2}$ Achilleas Tsoumanis, ${ }^{4}$ Bruno Meessen, ${ }^{1}$ \\ Raffaella Ravinetto ${ }^{1}$
}

To cite: Van Assche $K$, Nebot Giralt A, Caudron JM, et al. Pharmaceutical quality assurance of local private distributors: a secondary analysis in 13 low-income and middle-income countries. BMJ Glob Health 2018;3:e000771. doi:10.1136/ bmjgh-2018-000771

Handling editor Seye Abimbola

- Additional material is published online only. To view please visit the journal online (http://dx.doi.org/10.1136/ bmjgh-2018-000771)

Received 13 February 2018 Revised 1 May 2018 Accepted 2 May 2018

Check for updates

${ }^{1}$ Public Health Department, Institute of Tropical Medicine, Antwerp, Belgium ${ }^{2}$ QUAMED Quality Medicines for All, Castres, France ${ }^{3}$ Independent Researcher, Brussels, Belgium ${ }^{4}$ Clinical Sciences Department, Institute of Tropical Medicine, Antwerp, Belgium

\section{Correspondence to} Kerlijn Van Assche; vanassche.kerlijn.pharma@ gmail.com

\section{ABSTRACT \\ Introduction The rapid globalisation of the pharmaceutical production and distribution has not been supported by harmonisation of regulatory systems worldwide. Thus, the supply systems in low-income and middle-income countries (LMICs) remain exposed to the risk of poor-quality medicines. To contribute to estimating this risk in the private sector in LMICs, we assessed the quality assurance system of a convenient sample of local private pharmaceutical distributors.}

Methods This descriptive study uses secondary data derived from the audits conducted by the QUAMED group at 60 local private pharmaceutical distributors in 13 LMICs We assessed the distributors' compliance with good distribution practices (GDP), general quality requirements (GQR) and cold chain management (CCM), based on an evaluation tool inspired by the WHO guidelines 'Model Quality Assurance System (MQAS) for procurement agencies'. Descriptive statistics describe the compliance for the whole sample, for distributors in sub-Saharan Africa (SSA) versus those in non-SSA, and for those in lowincome countries (LICs) versus middle-income countries (MICs).

Results Local private pharmaceutical distributors in our sample were non-compliant, very low-compliant or lowcompliant for GQR (70\%), GDP (60\%) and CCM (41\%). Only $7 / 60$ showed good to full compliance for at least two criteria. Observed compliance varies by geographical region and by income group: maximum values are higher in non-SSA versus SSA and in MICs versus LICs, while minimum values are the same across different groups. Conclusion The poor compliance with WHO quality standards observed in our sample indicates a concrete risk that patients in LMICs are exposed to poor-quality or degraded medicines. Significant investments are needed to strengthen the regulatory supervision, including on private pharmaceutical distributors. An adapted standardised evaluation tool inspired by the WHO MQAS would be helpful for self-evaluation, audit and inspection purposes.

\section{INTRODUCTION}

Medicines must be of assured quality, for preventing harm to individual and public

\section{Key questions}

What is already known?

- Many medicine regulatory authorities in low-income and middle-income countries (LMICs) lack the resources needed to enforce full compliance with adequate quality standards.

- The quality systems of pharmaceutical distributors in the public sector in LMICs present important weaknesses.

What are the new findings?

- The quality systems of private pharmaceutical distributors in LMIC also appear to be poorly compliant with adequate quality standards, as defined by WHO.

What do the new findings imply?

- To prevent the distribution of poor-quality medicines, adequate quality systems should be enforced both in the public and private sectors.

- A standardised evaluation tool would improve the training, self-evaluation and regulatory inspection of pharmaceutical distributors in the private sector.

health, and for allowing an adequate performance of health systems. ${ }^{1}$ Unfortunately, the globalisation of pharmaceutical production and distribution has not been accompanied by a strengthening and harmonisation of the regulatory systems worldwide, and the global market is currently characterised by a situation of multiple pharmaceutical quality standards. ${ }^{1-4}$ Even if no country can be considered exempt from the risk of poor-quality medicines, problems abound especially in low-income and middle-income countries (LMICs), ${ }^{5}$ where many National Medicines Regulatory Authorities (NMRAs) lack the resources and capacity needed to enforce compliance with adequate quality standards. ${ }^{6-8}$

Poor-quality medicines include falsifications, which are deliberately or fraudulently 
misrepresented with regards to identity, composition or source, and substandards, which are authorised by the NMRA but fail to meet national and/or international standards, due to poor manufacturing and quality control practices that are not detected by the regulators. ${ }^{9}$ Poorquality medicines are well documented in the scientific literature. For instance, Nayyar and colleagues reported significant prevalence of low-quality medicines in 7 countries in Southeast Asia and in 21 countries in sub-Saharan Africa (SSA). ${ }^{10}$ A further systematic review showed a median prevalence of poor-quality anti-infective medicines in Africa and Asia of 28.5\%. ${ }^{11}$ Johnston and colleagues showed that substandard medicines are widespread in low-income countries (LICs) and can inadvertently lead to healthcare failure. ${ }^{12}$ More recent findings point at the presence of poor-quality medicines in the field of non-communicable diseases ${ }^{13-15}$ and in non-African settings, ${ }^{16-21}$ feeding the increasing awareness that poor-quality medicines are an important threat to individual and public health in LMICs. ${ }^{22}$ At the end of 2017, WHO estimated the rate of substandard and falsified medical products in LMICs at approximately $10.5 \% .^{23}$

In addition to being substandard or falsified from the manufacturing stage, medicines may become of poor quality because they degrade due to inappropriate storage and transport conditions along the supply chain ${ }^{1}$ : the inappropriate exposure to oxygen, moisture, heat and strong light may cause or accelerate the decomposition of the active or non-active ingredients and the formation of degradation products. ${ }^{24}$ To prevent such phenomena, all actors along the supply chain should implement the Good Distribution Practices (GDP $)^{25}$ as part of their quality system, and the compliance with GDP should be verified and enforced by the NMRA. ${ }^{26}$ Conversely, lack of stringent regulatory supervision will result in poor storage and distribution standards, triggering the presence of degraded medicines at the level of the concerned distributor(s), and downstream along the supply chain.

The WHO guideline 'Model Quality Assurance System (WHO MQAS) for procurement agencies', initially designed for United Nations agencies, may be referred to by any organisation that needs to design, implement or evaluate adequate quality systems in procurement and distribution of medicines. ${ }^{27}$ Our group previously contributed to unveil the vulnerabilities of the international pharmaceutical supply chains in the public health sector. In particular, our previous findings published by Nebot Giralt $e t a t^{28}$ suggest that international humanitarian distributors and SSA national procurement agencies do not consistently apply stringent quality criteria for selecting the medicines they supply: data from our sample showed that the compliance with the quality requirements of the WHO MQAS was generally low.

In LMICs, however, many patients are accessing healthcare through the private sector. ${ }^{29}$ It has even been suggested that the private sector could successfully substitute the public sector as healthcare provider because of the failures observed in the public health services. ${ }^{30} 31$
Therefore, we carried out a second study to evaluate the quality systems of pharmaceutical distributors in LMICs, this time with exclusive focus on the private sector. We assessed the performance of a convenient sample of private distributors in 13 LMICs by using an evaluation tool adapted from the WHO MQAS. Noteworthy, for this analysis we adopted a pragmatic definition of 'local private pharmaceutical distributors', that is, 'for-profit suppliers of medicines, not directly selling to patients, and selling locally in the country, or in a given area within the country'.

\section{METHODS}

This is a descriptive study of secondary data, conducted within the framework of a thesis of the Master's Course in International Health of the Institute of Tropical Medicine, Berlin, ${ }^{32}$ and complementary research at the Institute of Tropical Medicine, Antwerp. ${ }^{33}$ We assessed the compliance with MQAS principles of the quality systems of a convenient sample of local private pharmaceutical distributors in 13 LMICs.

Based on our previous findings,${ }^{28}$ and on publicly available information about the weakness of regulatory enforcement in many LMICs, ${ }^{78} 26$ we expected that the compliance with the principles of the WHO MQAS would be generally low. In addition, we hypothesised that the level of MQAS compliance will vary depending on those contextual factors that may have an impact on the stringency of regulatory supervision, such as the geographical localisation or a country's income group. Therefore, we also compared the WHO MQAS compliance of local private distributors in SSA versus those in non-SSA, and of those in LICs versus those in middle-income countries (MICs).

\section{Data collection and sampling}

Secondary data were derived from the database of QUAMED (Quality Medicines for all; https://quamed. org/en/home.aspx), a partnership that brings together medical non-governmental organisations active in LMICs and some African procurement agencies, and pleads for universal access to quality-assured medicines. ${ }^{28} 3435$ To help partner organisations purchasing quality-assured medicines, QUAMED evaluates the performance of various pharmaceutical suppliers, ${ }^{28}$ including local private distributors in the countries where partner organisations run their medical programmes.

Suppliers are audited by QUAMED if they (i) are current or potential suppliers of QUAMED partners and (ii) if they voluntarily undergo the audit, under a confidentiality agreement. Audits are conducted by a pool of qualified pharmacists, whose skills and qualifications are defined by a standard operating procedure. The audits' methodology varies depending on the categories of suppliers: big procurement agencies are audited according to the MQAS criteria ('full audit'), whereas local private distributors undergo a 'short audit', that is, 


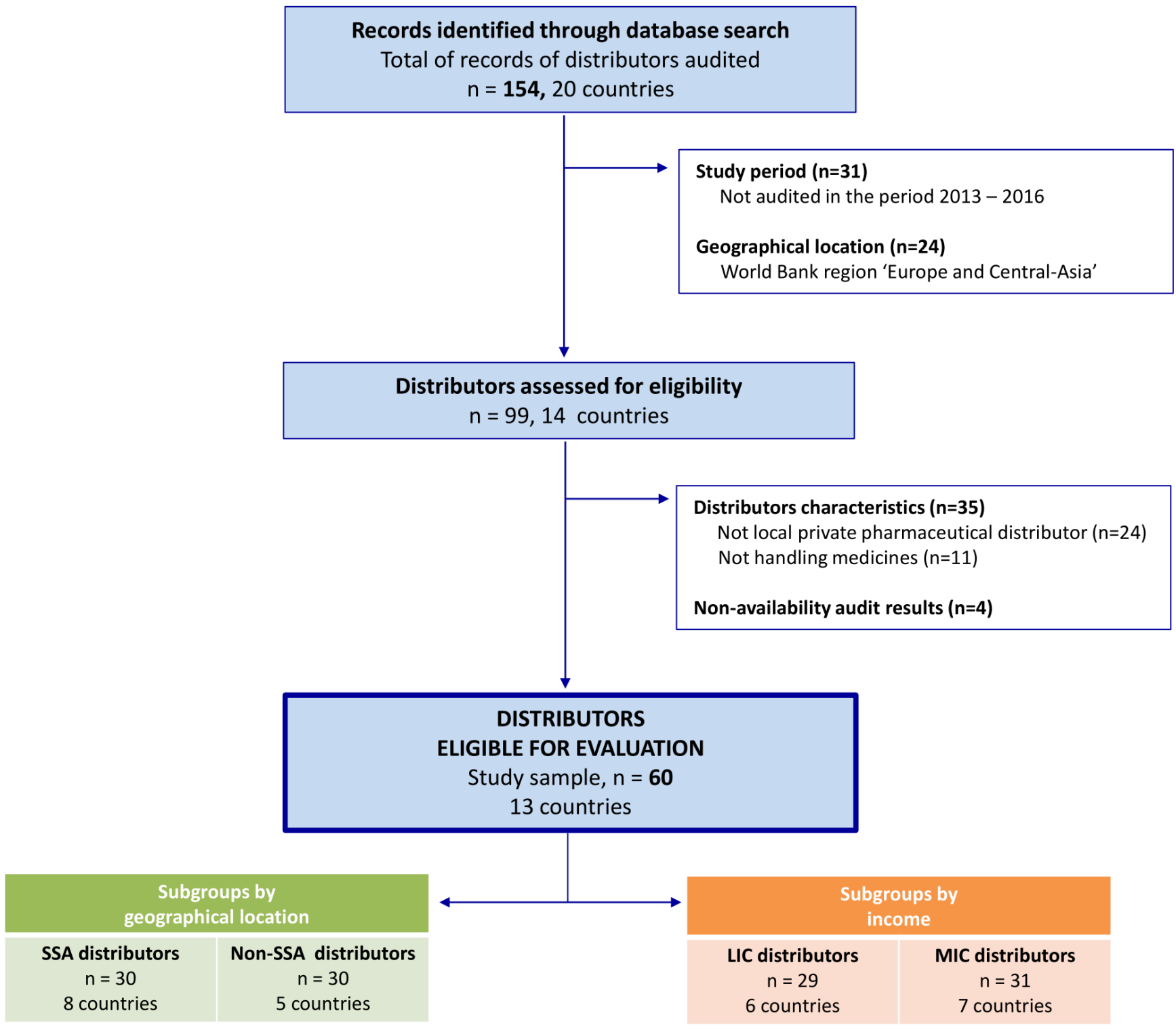

Figure 1 Flow diagram of the distributors' selection process. LIC, low-income countries, MIC, middle-income countries; n, number of distributors; SSA, sub-Saharan Africa.

a simplified evaluation inspired by the MQAS principles and focusing on GDP and quality systems. The 'short audit report' consists of a brief narrative and of a standardised questionnaire, which is a light version of the 'Harmonised MQAS Compliance Self-Assessment Tool'. ${ }^{36}$ Since audits are conducted under a confidentiality agreement, the reports are available to the QUAMED partners through a password-protected database.

The 'local private distributors' included in the present analysis were extracted from the QUAMED database according to the following criteria (see figure 1):

1. Period: conducted between 1January 2013 and 31 December 2016.

2. Geographical location: all countries where QUAMED has been doing audits, except the 2016 World Bank (WB) region 'Europe and Central-Asia'.
3. Type of distributor: local, for-profit pharmaceutical suppliers, handling medicines and not selling directly to patients.

4. Type of audit: QUAMED short audits, for which a short report including the standardised questionnaire is available.

For the comparison by subgroup, the distributors were classified as SSA or non-SSA by geographical region, and as MICs or LICs based on the WB classification (2016).

\section{Translation of the data}

For accurately comparing the audits' findings across distributors, we translated the narrative information contained in the 'short audit reports' (ie, a narrative text, and the open-ended answers of the standardised questionnaire), into quantitative data. In the above-mentioned 
Table 1 The five quality assurance criteria grouping the 12 activities derived from the Model Quality Assurance System

\section{Twelve activities derived from the Model Quality Assurance System}

Five quality assurance criteria

\begin{tabular}{lll}
\hline $1 \quad \begin{array}{l}\text { General quality } \\
\text { requirements (GQR) }\end{array}$ & $\begin{array}{l}\text { Documentation system } \\
\text { Human resources } \\
\text { Recall and complaints handling } \\
\text { Self-inspection }\end{array}$ \\
& \\
\hline $\begin{array}{l}\text { Good distribution } \\
\text { practices (GDP) }\end{array}$ & $\begin{array}{l}\text { Organisation of the warehouse } \\
\text { Physical storage conditions } \\
\text { Stock control } \\
\text { Control at reception } \\
\text { Distribution }\end{array}$ \\
\hline
\end{tabular}

3 Cold chain management (CCM) Management of the cold chain

5 Quality control (QC)

Quality control

$4 \quad$ Selection of sources (SS) Selection of the sources

\section{Definition}

GQR includes the basic elements required for the establishment of a quality assurance system which is effective in preventing mistakes.

For example: documentation system with standardised norms and procedures that define each activity of the quality assurance system, the management of human resources, and the recall and complaints handling.

GDP includes the process of reception, storage and distribution of medicines.

For example: it defines under what physical conditions the products must be stored (temperature, humidity...) to prevent their degradation (development of degradation products, loss of bioavailability, etc).

CCM includes all of the means used to ensure a predefined temperature (generally between $+2^{\circ} \mathrm{C}$ and $+8^{\circ} \mathrm{C}$ ) for temperature-sensitive products as they move through the supply chain, and so to ensure their quality, efficacy and safety.

$S S$ includes the criteria adopted to choose products from manufacturers that comply with good manufacturing practices. It is taken as an indicator of the quality assurance of the products as supplied by the manufacturers.

$Q C$ includes the design and implementation of a sampling plan to check the quality of supplied products, through full chemical analysis conducted at an accredited laboratory. work, Nebot Giralt et al developed a 'rating system' based on the key activities of the WHO MQAS and used it to measure the MQAS compliance of pharmaceutical procurement agencies undergoing the QUAMED 'full audits'. ${ }^{28}$ We adapted this tool, to capture the information collected during the QUAMED 'short audits' of local private pharmaceutical distributors, and we developed an $a d$ hoc 'rating system for local private pharmaceutical distributors' (online supplementary materials). This is based on 12 activities, derived from the WHO MQAS and grouped into five quality assurance (QA) criteria: general quality requirements (GQR), GDP, cold chain management (CCM), selection of sources (SS) and quality control (QC). Details and examples of such activities and criteria are given in table 1.

To analyse our data, we first translated the narrative information contained in the 'short audit reports' into a numerical value (score) per activity. Second, we rated each distributor for each of the 12 activities by attributing a score between 0 and 4 , where each number represents a specific level of compliance. Third, we calculated the compliance of each distributor with each of the five QA criteria by calculating the average of the scores of the activities included in each criterion (raw data available as online supplementary materials). For both 'activities' and 'criteria', the score that expresses the level of compliance with the principles of MQAS ranges from non-compliance $(0.00)$ to consistently compliant (4.00). Intermediate scores are very low compliance (0.01-0.99), low compliance (1.00-1.99), medium compliance (2.002.99) and good compliance (3.00-3.99).

Even if the 'rating system for local private pharmaceutical distributors' includes five QA criteria, in this paper we only report on findings for GQR, GDP and CCM, which represent the core business of all pharmaceutical distributors. As further elaborated in the discussion section, the two additional criteria (SS and $\mathrm{QC}$ ) refer to prequalification and reassessment of procured sources, for which local private distributors rely on the marketing authorisation granted by the NMRA.

\section{Analysis}

The quantitative data reported in the 'rating system for local private pharmaceutical distributors' were analysed using Microsoft Excel 2007. The units of analysis were the local private pharmaceutical distributors. The variables were the three QA criteria (GQR, GDP and CCM), treated as ordinal variables. General descriptive statistics 
were performed to identify the level of compliance of the distributors with each of the three criteria (median, IQR, minimum and maximum values). The data were analysed for the full sample and by subgroups (i) prespecified by geographical region: SSA versus non-SSA and (ii) exploratory by WB economic classification: LICs versus MICs. Internal consistency of the criteria GQR and GDP was evaluated with Cronbach's $\alpha$ to measure whether the different activities that are meant to measure the same criterion produce similar scores.

Primary data were collected by QUAMED under a confidentiality agreement with the audited suppliers; therefore, in order to ensure confidentiality, the identity of the individual distributors will not be disclosed.

\section{RESULTS}

Overall, 154 records of audited distributors were identified through the QUAMED database search. The flow diagram of the distributors' selection process is shown in figure 1 . The records of 60 distributors in 13 countries (ie, Burkina Faso, Cameroon, the Democratic Republic of Congo, Ethiopia, Haiti, Ivory Coast, Jordan, Kenya, Lebanon, Myanmar, Niger, the Philippines and Senegal) were eligible for inclusion in the analysis. Out of these 60 distributors, 30 are located in SSA (eight countries) and 30 outside SSA (five countries); 29 are located in LICs (six countries) and 31 in MICs (seven countries).

\section{Compliance with QA criteria in LMICs}

The compliance with the QA criteria varies strongly across distributors (table 2). The internal consistency is high for GDP $(\alpha=0.87)$ and GQR $(\alpha=0.88)$. For these criteria, compliance is generally low, with central tendency scores with a median of 1.13 for GQR (IQR 0.50-2.00) and 1.50 for GDP (IQR 0.80-2.25). Out of 60 distributors included in the analysis, only 44 supplied heat-sensitive products and were evaluated for CCM. In general, compliance was better for CCM than for other QA criteria, with a central tendency of 2.00 (IQR 1.00-3.00). However, CCM also showed the highest variability, with scores ranging from non-compliant (0) to fully compliant (4). Coded data by distributor are available in the online supplemental material.

The percentage of distributors found to be non-compliant, very low-compliant or low-compliant with the QA criteria is of $70 \%$ for GQR, $60 \%$ for GDP and $41 \%$ for CCM (figure 2). The compliance was on average lower for GQR than for GDP. All distributors showed some level of non-compliance with the QA criteria, and only seven distributors out of 60 showed good to full compliance for at least two criteria. None of the distributors was fully compliant with GQR or GDP criteria.

\section{Compliance with QA criteria by geographical region and by income group}

The internal consistency of GDP and GQR was high for the geographical subgroups (GDP: SSA $\alpha=0.80$, non-SSA $\alpha=0.90$ and GQR: SSA $\alpha=0.80$, non-SSA $\alpha=0.87$ ) and for the income subgroups (GDP: LIC $\alpha=0.78$, MIC $\alpha=0.90$ and GQR: LIC $\alpha=0.71$, MIC $\alpha=0.87$ ). The compliance with the three QA criteria varies by geographical region and by income group (Online Supplementary Table B). In particular, it is higher (low to medium) for non-SSA than for SSA (very low to low). Minimum values are the same in both groups, while the IQR and the maximum values are higher in non-SSA than in SSA (table 2); within non-SSA there is high variability across distributors, whereas in SSA the compliance is generally very low or low (figure 3 ).

Similar results are found when comparing the LICs versus MICs subgroups. Despite the pronounced differences between the two subgroups, the lower scores are the same for LICs and MICs (table 2). The criteria with the highest and lowest scores tended to be the same in all subgroups.

\section{DISCUSSION}

Our evaluation of the quality systems of a convenient sample of 60 private pharmaceutical distributors in 13 LMICs, conducted with an ad hoc rating system, is to the best of our knowledge the first study of this kind. Our results show a generally poor compliance with adequate

Table 2 Overview of the level of compliance of local private pharmaceutical distributors, by quality assurance criterion

\begin{tabular}{|c|c|c|c|c|c|c|c|}
\hline & \multirow{2}{*}{$\begin{array}{l}\text { Distributors } \\
\mathrm{N}\end{array}$} & \multicolumn{2}{|c|}{$\begin{array}{l}\text { General quality } \\
\text { requirements }\end{array}$} & \multicolumn{2}{|c|}{$\begin{array}{l}\text { Good distribution } \\
\text { practices }\end{array}$} & \multicolumn{2}{|c|}{ Cold chain management } \\
\hline & & Median (IQR) & Min-max & Median (IQR) & Min-max & Median (IQR) & Min-max \\
\hline Geographical region & $\mathrm{n}$ & & & & & & \\
\hline Sub-Saharan Africa & $30\left(20^{\star}\right)$ & $0.8(0.3-1.3)$ & $0.3-3.0$ & $1.0(0.6-1.8)$ & $0.0-2.6$ & $1.0(0.0-2.0)$ & $0.0-4.0$ \\
\hline Low-income countries & $29\left(20^{\star}\right)$ & $0.8(0.3-1.3)$ & $0.3-2.0$ & $1.0(0.6-1.6)$ & $0.0-2.6$ & $1.0(0.0-2.0)$ & $0.0-3.0$ \\
\hline Middle-income countries & $31\left(24^{\star}\right)$ & $2.0(0.9-2.5)$ & $0.3-3.4$ & $2.0(1.4-2.7)$ & $0.0-3.6$ & $2.0(2.0-3.3)$ & $0.0-4.0$ \\
\hline
\end{tabular}

The level of compliance was evaluated on a scale from 0 to 4.

${ }^{\star}$ Number of distributors supplying cold chain products. 


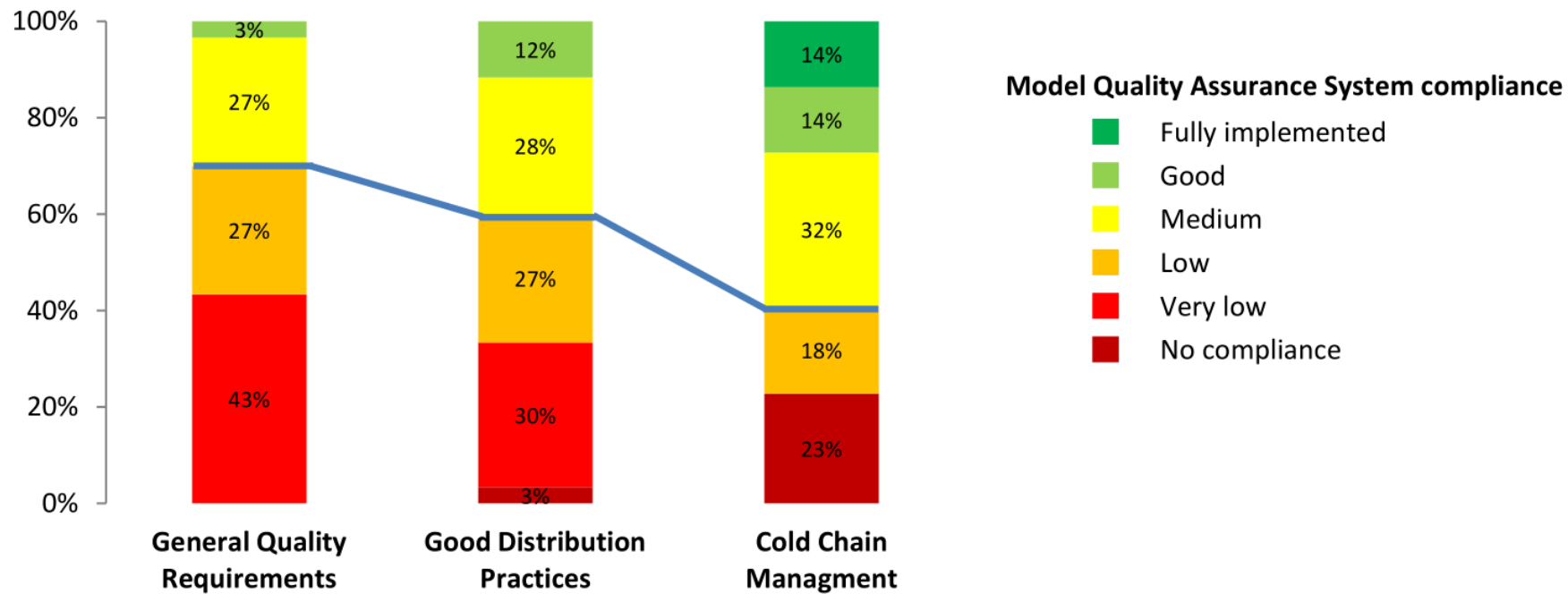

Figure 2 Percentage of local private pharmaceutical distributors in each level of compliance, for the full sample, for each quality assurance criterion. Frequency distribution. Quality assurance criteria: general quality requirements ( $n=60$ ), good distribution practices $(n=60)$ and cold chain management $(n=44)$. Below the blue line are the distributors with no compliance, very low compliance or low compliance, above the blue line are the distributors with medium, good or full compliance. $n$, number of distributors evaluated.

QA standards. This confirms our initial hypothesis that the compliance with the principles of the WHO MQAS would be generally low, and it is per se not surprising, given the weakness of regulatory oversight in many LMICs. $^{78} 26$ Our results also indicate a better (even though still low) compliance for distributors located outside SSA than for those in SSA, and for those located in MICs than for those in LICs. This is also per se not surprising: NMRAs in poorer countries are more likely to be unable to (fully) fulfil their core tasks, that is, ensuring the quality, efficacy and safety of all medicines circulating in their country, due to lack of adequate resources. ${ }^{26} 37-39$

We also noted an important in-country variability in our sample, that is, it may happen that a same NMRA licences both well-performing and poorly performing distributors. We may tentatively propose two explanations for this variability. First, it may be due to the unclear definition of the standards for storage and distribution in the national regulation since GDP are often not included in the pharmaceutical legislation of many countries. Or when reference to GDP is made, the standards are not adequately specified, for instance, in its assessment of medicines regulatory systems in 26 SSA countries, WHO reported in 2010 that national guidelines are often not in line with the WHO GDP standards. ${ }^{26}$ Second, it may be due to the poor or variable supervision on the pharmaceutical distributors by non-stringent NMRAs ${ }^{40}$ : in the same assessment of medicines regulatory systems in 26 SSA countries, ${ }^{26}$ WHO reported that the structures in place to inspect pharmaceutical distributors are often not well coordinated and that the quality of the inspections is limited. On a similar line, a situation analysis on effective management of medicines in 11 South East Asian countries showed limited capacity of NMRAs to carry out their tasks, including inspections, and advised to review and update the medicines regulation to ensure compliance with international standards. ${ }^{41}$ Irrespective of the specific cause, the variability of the quality systems across distributors in a country implies a variable level of QA, and a real risk of exposure to poor-quality or degraded medicines.

The observed variability also implies that, even if all the distributors in our sample are located in countries with no Stringent Regulatory Authority, ${ }^{39} 4142$ some of them may still achieve acceptable standards, with good to full compliance for two QA criteria out of three. It will be worthwhile to investigate the non-regulatory drivers of QA compliance; at this stage, we hypothesise that incentives to invest in quality systems may descend from a corporate policy, or they may come from the market, as previously suggested by McCabe and colleagues. ${ }^{43}$ In particular, if their main clients have strict quality requirements (or if they wish to reach such clients), distributors will invest to improve their QA system. At international level, the positive attitude of many manufacturers towards the WHO Prequalification Programme ${ }^{5}$ is a good example of such a market incentive. At local level, a distributor might decide to procure mainly or only medicines approved by Stringent Regulatory Authorities, either because it targets clients who ask such products and tries to serve this market 'niche', or because it tries to create a market demand, by differentiating its own offer from the competitors. ${ }^{44}$

However, as said above, our overall results show a generally poor compliance with adequate QA standards. To correct this situation, countries need to put in place stewardship of the pharmaceutical system as well as an adequate national pharmaceutical policy. ${ }^{45}{ }^{46}$ To do so, a 
General Quality Requirements

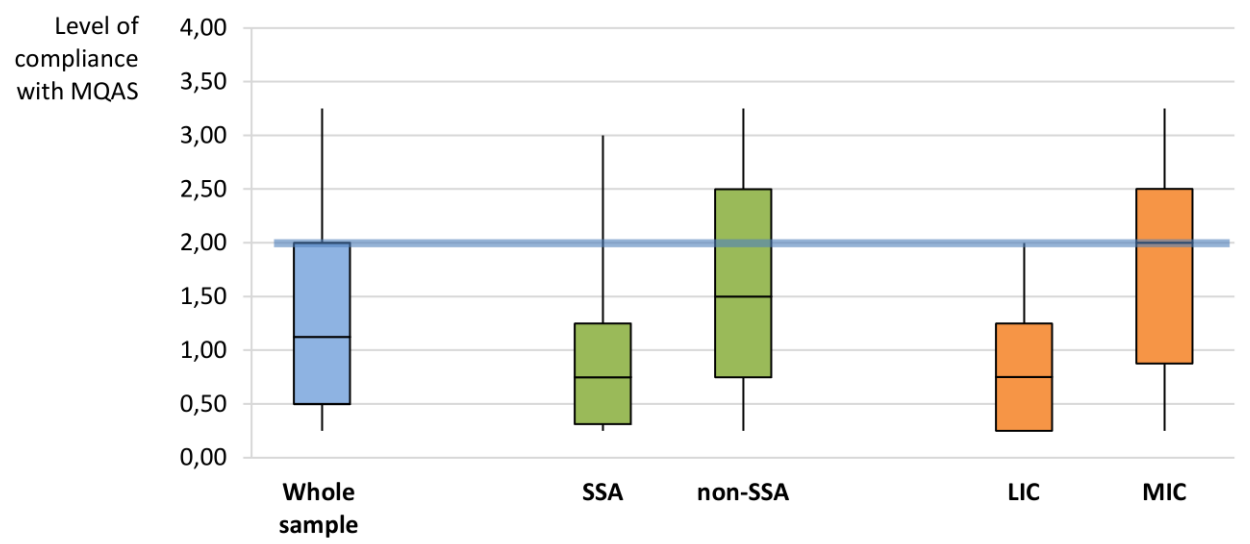

\section{Good Distribution Practices}

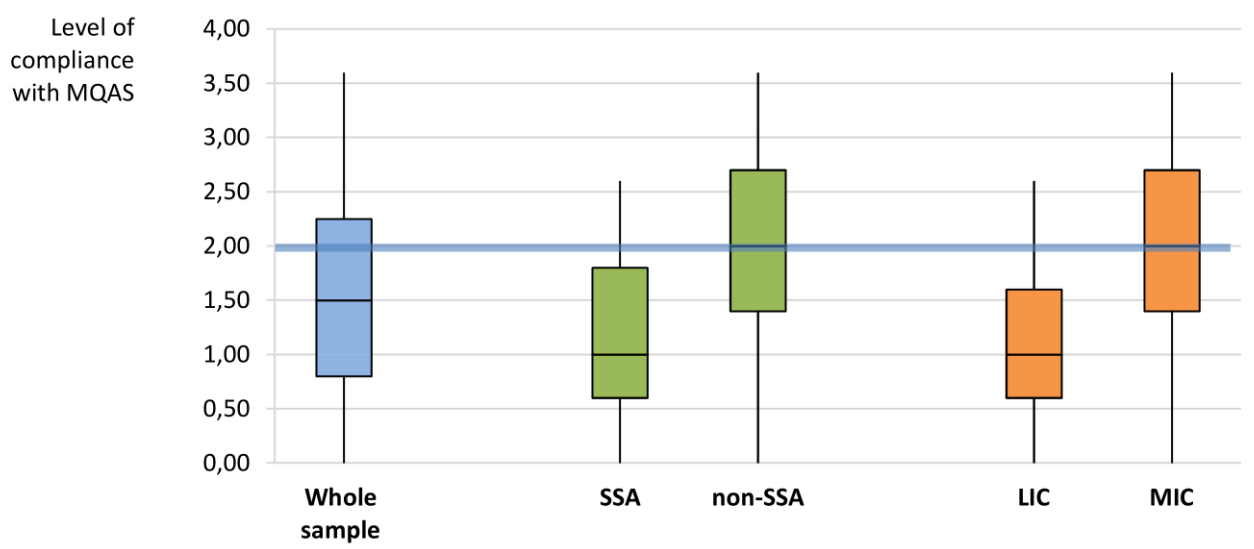

\section{Cold Chain Management}

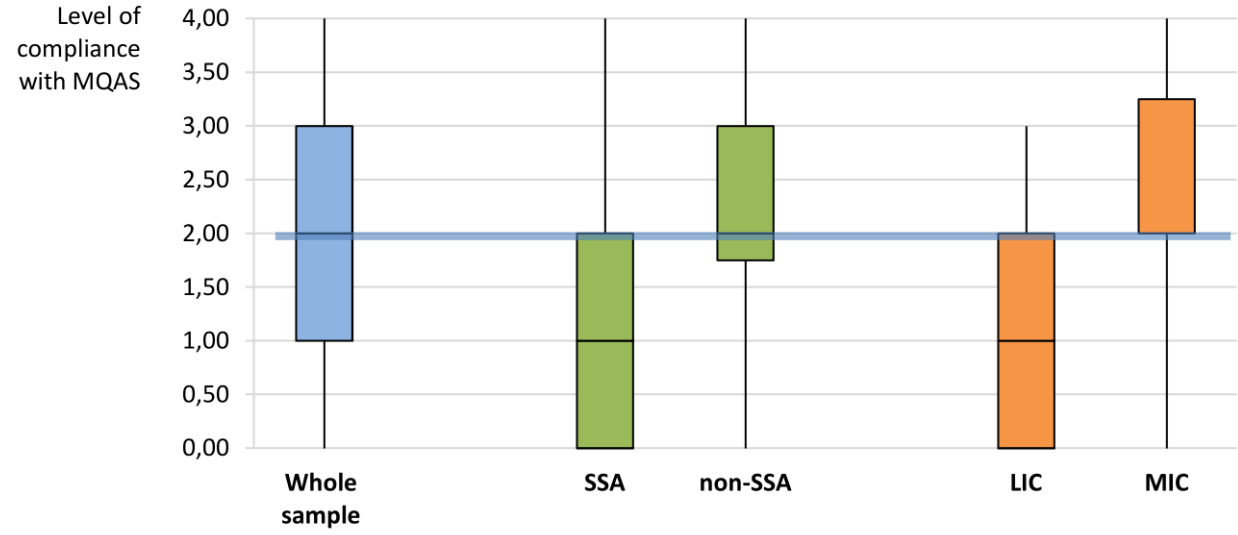

Figure 3 Level of compliance of the full and the subsamples of local private pharmaceutical distributors, by quality assurance criterion. Boxplot. Below the blue line are the distributors with no, very low or low compliance, above the blue line are the distributors with medium, good or full compliance. LIC, low-income country; MIC, middle-income country; MQAS, Model Quality Assurance System; SSA, sub-Saharan Africa.

strong commitment is needed at governmental level, ${ }^{47}$ by donors and by the private sector.

The 'rating system for local private pharmaceutical distributors' developed for this analysis is inspired by the WHO MQAS, ${ }^{27}$ a guideline that proved to be useful for the evaluation of national procurement centres and international humanitarian distributors. ${ }^{28}$ However, the present analysis suggests that the WHO MQAS is not fully applicable for local pharmaceutical distributors. In particular, even if our rating system included five QA criteria inspired by the WHO MQAS, in this paper we do not report on the criteria SS and QC, which correspond to the WHO MQAS chapters on prequalification and reassessment of procured sources. The reason for this choice is that in this case the WHO MQAS requirements exceed by far the mandate of local distributors, which are (like 
national procurement centres) 'only' legally required to procure and supply medicines that have been granted a marketing authorisation by the NMRA. ${ }^{48}$ In other words, their criterion for selecting sources is the registration granted (and confirmed over time) by the NMRA. If the NMRA is weak, there is an increased risk that medicines supplied by these distributors are poorly quality assured, not because of the distributors' deficiency, but because of the deficiencies of the regulatory environment in which they operate. The 'quality risk' remains real, but it would not be realistic (nor fair) to expect that local distributors set up a QA system including a stringent prequalification and reassessment system, to compensate the deficiencies of the NMRA.

Conversely, the criteria derived from the WHO MQAS for GQR, GDP and CCM are inherent to the mandate of any pharmaceutical distributor, and should be fully implemented. Unfortunately, our findings in this respect are in line with previous studies: in insufficiently regulated environments, the implementation of GDP does not seem to be a priority, ${ }^{43}$ and the storage conditions are often suboptimal. ${ }^{3849}$ In 2011, Dickens and colleagues had noted that distributors at peripheral level were unlikely to meet the model standards for premises, equipment or staffing in the near future. ${ }^{50}$ Other authors emphasised the scarcity of data from formal assessments of the quality systems along the medicines' distribution chain in LMICs. ${ }^{38} 49$ These findings are still an issue today. The WHO GDP guidelines serve as a reference guideline for good practices. However, to the best of our knowledge, there is no standardised assessment tool for evaluating the quality systems of local pharmaceutical distributors, through measurable variables, that allows for scores' comparisons across countries and regions (research objective), and for a given distributor over time (monitoring and evaluation objective). Such a practical, harmonised tool would be useful for self-assessment, for regulatory supervision and for monitoring and training purposes.

Since the current situation of poor compliance with adequate quality standards is largely due to poor regulation and/or poor regulatory enforcement in LMICs (in addition to important concomitant factors, such as lack of market incentives for quality and a lack of benchmarking, especially in-country), it would be also worthwhile to assess the impact of improved regulatory policies on the performance of pharmaceutical distributors. For instance, Ethiopia has recently published its official GDP guidelines, ${ }^{51}$ and it could be interesting to formally assess their impact on distributors' practices after a few years.

This work has several limitations. First, we used a convenient sample and no statistical tests were performed. Second, narrative reports were retrospectively translated into quantitative scores by a researcher who had not taken part in the audits. Third, our findings may be positively biased by the fact that distributors included in our samples voluntarily agreed to undergo the audit, which may indicate a positive attitude towards quality improvement. On a minor note, the fact that our findings for the criterion CCM were better than for GQR and GDP can be biased by the fact that only part of the distributors in our sample handled heat-sensitive products, and they were often those with better GDP compliance. (In addition, in the primary data we could not capture all the information that would be needed to fully assess the quality of CCM.) Our findings should ideally be confirmed by further research, with prospective collection of quantitative data in the standardised rating system. Furthermore, qualitative research would be needed to shed light about drivers or hindering factors for compliance with GDP. Nevertheless, our results are based on an evaluation of 60 different distributors in 13 countries, so the observed poor compliance with QA criteria remains alarming.

Some authors have suggested that the private sector could successfully substitute the public sector in the provision of healthcare in LMICs, ${ }^{30}{ }^{43}$ while others suggested on different grounds that the private sector is not a valuable alternative for healthcare and services, and that privatisation may have negative implications for affordability, socioeconomic determinants of use and quality ${ }^{30}$ In the previous study on the QUAMED database, Nebot Giralt et $a l^{28}$ found that compliance with the MQAS of African pharmaceutical distributors in the public and not-for-profit sector was low. But our study now shows at least comparable weaknesses in the private sector. Overall, our findings combined with those of Nebot Giralt $e t a l^{28}$ are in line with the view of those who contend that governments, as the steward per se within the health system, should not concentrate their efforts on the improvement of a particular sector (public or private), but should understand and supervise the health system as a whole..$^{38} 5253$ This applies also to the strengthening of the pharmaceutical sector and pharmaceutical supply system, either public or private.

\section{CONCLUSION}

This study investigated the quality systems of local, private pharmaceutical distributors in LMICs, using a standardised tool derived from the WHO MQAS. Our findings suggest insufficient compliance with GQR and with GDP, implying a significant risk of exposure to poorquality or degraded medicines. Regulatory supervision on these distributors should be strengthened to ensure that compliance with stringent quality and GDP criteria becomes an essential prerequisite for being licensed. In addition, a positive market incentive towards quality would also trigger quality improvements. The development of a standardised evaluation tool inspired by the WHO MQAS but adapted to the characteristics of these distributors could be helpful for monitoring and evaluation, for regulatory inspection and as training tool to upgrade the current standards. These corrective measures should be framed in a more general strengthening of regulatory capacities in LMICs, including effective harmonisation initiatives. 
Twitter Follow Raffaella Ravinetto @RRavinetto, Follow Bruno Meessen @ bmeessen

Acknowledgements The authors thank all the distributors that accepted to be audited by QUAMED. They thank all QUAMED partners for contributing to the development of the network and making this project possible. They also thank Christophe Luyckx and Vera Melotte, the former coordinators of the QUAMED project. Finally, they thank Dr Matthias Borchert, who coached KVA during the Master in International Health.

Contributors Conceived and designed the survey: KVA, RR, ANG, JMC, BS and CP. Performed some of the audits: JMC and CP. Performed the survey and analysed the data: KVA. Wrote the paper: KVA and RR. Reviewed the paper: ANG, JMC, BS, CP, AT and BM.

Funding The audits described in this paper were funded by the ECHO program of the European Commission, the Belgian Development Cooperation and membership fees by QUAMED members. A part of the research work of KVA was funded by the Belgian Development Cooperation.

Competing interests None declared.

Ethics approval The study protocol was approved by the Institutional Review Board of the Institute of Tropical Medicine, Antwerp, Belgium (IRB/AB/ac/100_ref:1102/16; IRB/AB/ac/057_ref:1167/17).

Provenance and peer review Not commissioned; externally peer reviewed.

Data sharing statement Coded data are available in the article. Motivated request to access data by country should be addressed to the corresponding author.

Open Access This is an Open Access article distributed in accordance with the Creative Commons Attribution Non Commercial (CC BY-NC 4.0) license, which permits others to distribute, remix, adapt, build upon this work non-commercially, and license their derivative works on different terms, provided the original work is properly cited and the use is non-commercial. See: http://creativecommons.org/ licenses/by-nc/4.0/

(C) Article author(s) (or their employer(s) unless otherwise stated in the text of the article) 2018. All rights reserved. No commercial use is permitted unless otherwise expressly granted.

\section{REFERENCES}

1. Newton PN, Amin AA, Bird C, et al. The primacy of public health considerations in defining poor quality medicines. PLoS Med 2011;8:e1001139.

2. Caudron JM, Ford N, Henkens M, et al. Substandard medicines in resource-poor settings: a problem that can no longer be ignored. Trop Med Int Health 2008;13:1062-72.

3. Kuehn BM. As production goes global, drug supply faces greater risks to safety, quality. JAMA 2011;306:811-3.

4. Bate R, Zhe Jin G, Mathur A, et al. Poor quality drugs and global trade: a pilot study. NBER Working Paper Series 2014, Camebridge: National Bureau of Economic Research. http://www.nber.org/papers/ w20469.pdf (cited 6 Jan 2018).

5. 't Hoen EF, Hogerzeil HV, Quick JD, et al. A quiet revolution in global public health: the world health organization's prequalification of medicines programme. J Public Health Policy 2014;35:137-61.

6. World Health Organisation. Norms and standards: 70 years of WHO standards on medicines quality, expert committee on specifications for pharmaceutical preparations 1947-2017: addressing changing public health challenges. WHO Drug Information 2017;31:15-26.

7. World Health Organisation. WHO regulatory harmonization: updating medicines regulatory systems in sub-Saharan African countries. WHO Drug Information 2010;24:6-20.

8. Preston C, Valdez ML, Bond K. Strengthening medical product regulation in low- and middle-income countries. PLoS Med 2012;9:e1001327.

9. World Health Organisation. Substandard and falsified medical products. 70th World Health Assembly; 2017 22-31 May; Geneva, Switzerland. http://www.who.int/mediacentre/news/releases/2017/ dementia-immunization-refuguees/en/ (cited 5 Nov 2017).

10. Nayyar GM, Breman JG, Newton PN, et al. Poor-quality antimalarial drugs in southeast Asia and sub-Saharan Africa. Lancet Infect Dis 2012;12:488-96.

11. Almuzaini T, Choonara I, Sammons H. Substandard and counterfeit medicines: a systematic review of the literature. BMJ Open 2013;3:e002923.
12. Johnston A, Holt DW. Substandard drugs: a potential crisis for public health. Br J Clin Pharmacol 2014;78:218-43.

13. Antignac M, Diop BI, Macquart de Terline D, et al. Fighting fake medicines: first quality evaluation of cardiac drugs in Africa. Int $J$ Cardiol 2017;243:523-8.

14. Mumphansha H, Nickerson JW, Attaran A, et al. An analysis of substandard propofol detected in use in Zambian Anesthesia. Anesth Analg 2017;125:616-9.

15. Torloni MR, Gomes Freitas C, Kartoglu UH, et al. Quality of oxytocin available in low- and middle-income countries: a systematic review of the literature. BJOG 2016;123:2076-86.

16. Medina E, Bel E, Suñé JM. Counterfeit medicines in Peru: a retrospective review (1997-2014). BMJ Open 2016;6:e010387.

17. Hetzel MW, Page-Sharp M, Bala N, et al. Quality of antimalarial drugs and antibiotics in Papua New Guinea: a survey of the health facility supply chain. PLoS One 2014;9:e96810.

18. Tabernero $P$, Mayxay $M$, Culzoni MJ, et al. A repeat random survey of the prevalence of falsified and substandard antimalarials in the Lao PDR: a change for the better. Am J Trop Med Hyg 2015;92(6 Suppl):95-104.

19. Guo S, Kyaw MP, He L, et al. Quality testing of artemisininbased antimalarial drugs in Myanmar. Am J Trop Med Hyg 2017:97:1198-203

20. Pan $\mathrm{H}$, Luo $\mathrm{H}$, Chen $\mathrm{S}$, et al. Pharmacopoeial quality of antimicrobial drugs in southern China. Lancet Glob Health 2016;4:e300-2.

21. Khojah HMJ, Pallos H, Yoshida N, et al. The quality of medicines in community pharmacies in Riyadh, Saudi Arabia: A Lot Quality Assurance Sampling (LQAS)-based survey. Pharmacology Pharmacy 2013;04:511-9.

22. Ravinetto $R$, Vandenbergh $D$, Macé $C$, et al. Fighting poor-quality medicines in low- and middle-income countries: the importance of advocacy and pedagogy. J Pharm Policy Pract 2016;9:36.

23. World Health Organization. WHO global surveillance and monitoring system for substandard and falsified medical products. Geneva: World Health Organisation, 2017.

24. Hodel EM, Kaur H, Terlouw DJ. Stability of dihydroartemisininpiperaquine tablet halves during prolonged storage under tropical conditions. Am J Trop Med Hyg 2017;96:338-40.

25. World Health Organisation. Annex 5: WHO good distribution practices for pharmaceutical products', WHO technical report series 2010. 957:235-64.

26. World Health Organisation. Assessment of medicines regulatory systems in sub-Saharan African countries. 2010 http://apps.who.int/ medicinedocs/en/d/Js17577en/ (cited 11 Nov 2017).

27. World Health Organisation. Model quality assurance system for procurement agencies. In: Annex III of the WHO technical report series 986: WHO Expert Committee on Specifications for Pharmaceutical Preparations, forty-eighth report. 2014 http://apps. who.int/medicinedocs/en/d/Js21492en/ (cited 11 Nov 2017).

28. Nebot Giralt A, Schiavetti B, Meessen B, et al. Quality assurance of medicines supplied to low-income and middle-income countries: poor products in shiny boxes? BMJ Glob Health 2017;2:e000172.

29. Mills $A$. Health care systems in low- and middle-income countries. $N$ Engl J Med 2014;370:552-7.

30. Oxfam International. Blind optimism: challenging the myths about private health care in poor countries. Oxfam Briefing Paper 2009; 125: 1-55. https://policy-practice.oxfam.org.uk/publications/blindoptimism-challenging-the-myths-about-private-health-care-in-poorcountries-114093

31. McCabe A, Seiter A, Diack A, et al. Private sector pharmaceutical supply and distribution channels in Africa, Health, Nutrition and Population (HNP) discussion paper 2011, Washington DC: World Bank. https://openknowledge.worldbank.org/bitstream/handle/ 10986/13590/656010WP00PUBL00PvtSectorPharma0811.pdf? sequence $=1$ \&isAllowed $=y$ (cited 7 April 2018).

32. Van Assche K. The quality of medicines of the private pharmaceutical distributors in sub-Saharan Africa: a secondary quantitative data analysis from a sample of private distributors, International Health [thesis], Berlin: Charité - Universitätsmedizin. 2016. rravinetto@itg.be

33. Van Assche K. The quality of medicines supplied by private pharmaceutical distributors in non-Sub-Sahara African low- and middle-income countries [research report], Antwerp, Institute of Tropical Medicine. 2017 rravinetto@itg.be

34. Caudron J, Luyckx C, Ravinetto R. Quamed: a North-South collaborative approach toward universal access to quality medicines. In: 60th Meeting of the American Society of Tropical Medicine and Hygiene. Philadelphia, 2011.

35. Moore T, Lee D, Konduri N, et al. Assuring the quality of essential medicines procured with donor funds. Health, nutrition and population discussion paper 2012; Washington DC: World Bank. 
https://openknowledge.worldbank.org/handle/10986/13577 (cited 11 Nov 2017).

36. World Health Organization. Medicines quality assurance: a harmonized self-assessment tool for procurement agencies. WHO Drug Information 2014;28:434-47.

37. Ratanawijitrasin S, Wondemagegnehu E. Effective drug regulation: a multicountry study. Geneva: World Health Organisation. 2002 http:// apps.who.int/iris/bitstream/10665/42470/1/9241562064.pdf (cited 11 Nov 2107).

38. Yadav P, Tata HL, Babaley M. The world medicines situation-storage and supply chain management. 3rd edn. Geneva: World Health Organisation, 2011.

39. World Health Organization. International harmonization, WHO drug information. , 2008:22, 177-218.

40. WHO Prequalification Team. Clarification with respect to a stringent regulatory organization as applicable to the stringent regulatory authority guideline [Internet], Guidance document. 2017 https:// extranet.who.int/prequal/sites/default/files/documents/75\%20SRA\% 20clarification_February2017_0.pdf (cited 10 Oct 2017).

41. World Health Organization. General effective management of medicines, report of the South-East Asia regional consultation. New Delhi: World Health Organization, Regional Office for SouthEast Asia, 2013. http://apps.who.int/medicinedocs/documents/s21587en/ s21587en.pdf (cited 7 Apr 2018)

42. World Health Organisation. Definition of stringent regulatory authority. WHO technical report series 1003. WHO Expert Committee on Specifications for Pharmaceutical Preparations, fifth first report. 2017 http://www.who.int/medicines/areas/quality_safety/ quality_assurance/expert_committee/WHO_TRS_1003_full-version. pdf?ua=1 (cited 6 Apr 2018).

43. McCabe A. Private sector pharmaceutical supply and distribution chains: Ghana, Mali and Malawi. Health Systems for Outcomes Publication. Washington: World Bank, 2009. http://documents. worldbank.org/curated/en/745621468270302773/pdf/531070WP OPharm10Box345597B01PUBLIC1.pdf (cited 11 Nov 2017).

44. Mackintosh M, Chaudhuri S, Mujinja PG. Can NGOs regulate medicines markets? Social enterprise in wholesaling, and access to essential medicines. Global Health 2011;7:4.
45. Pezzola A, Sweet CM. Global pharmaceutical regulation: the challenge of integration for developing states. Global Health 2016;12:1-18.

46. Seiter A. Pharmaceuticals: drug regulation in low and middle income countries. health, nutrition and population Brief \#4; Washington DC: World Bank. 2005 http://documents.worldbank.org/curated/en/ 954371468194045308/pdf/321940HNPBrief140DrugOregulation.pdf (cited 11 Nov 2017).

47. Trap B, Kikule K, Vialle-Valentin C, et al. First regulatory inspections measuring adherence to good pharmacy practices in the public sector in Uganda: a cross-sectional comparison of performance between supervised and unsupervised facilities. J Pharm Policy Pract 2016;9:1-10.

48. Committee on Understanding the Global Public Health Implications of Substandard, Falsified, and Counterfeit Medical Products. In: Buckley G, Gostin L, eds. Countering the problem of falsified and substandard drugs. Washington (DC): National Academies Press, 2013.

49. Dowling P. Healthcare supply chains in developing countries: situational analysis. Arlington (VA): USAID 2011. deliver project Task Order 4. http://pdf.usaid.gov/pdf_docs/PA00MKKG.pdf (cited 11 Nov 2017).

50. Dickens T. The world medicines situation-procurement of medicines 3rd edn. Geneva: World Health Organisation, 2011.

51. Food, medicine and health care administration and control authority of Ethiopia. Guidelines for: good storage practices, good distribution practice, pharmaceutical product recall. 1st ed. Addis abeba: EFMHACA. 2015 http://www.fmhaca.gov.et/documents/GDP,\% 20GSP\%20and\%20Recale\%20Guideline\%202015 (cited 7 Apr 2017).

52. McPake B, Hanson K. Managing the public-private mix to achieve universal health coverage. Lancet 2016;388:622-30.

53. da Fonseca EM. How can a policy foster local pharmaceutical production and still protect public health? Lessons from the health-industry complex in Brazil. Glob Public Health 2018;13:489-502. 